\begin{tabular}{|c|c|}
\hline InTSRD & $\begin{array}{l}\text { International Journal of Trend in Scientific Research and Development (IJTSRD) } \\
\text { International Open Access Journal | www.ijtsrd.com }\end{array}$ \\
\hline 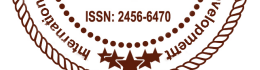 & ISSN No: 2456 - 6470 | Volume - 2 | Issue - 6 | Sep-Oct 2018 \\
\hline
\end{tabular}

\title{
Nil Complementary Domination in Intutionistic Fuzzy Graph
}

\author{
R. Buvaneswari ${ }^{1}$, K. Jeyadurga ${ }^{2}$ \\ ${ }^{1}$ Assistant Professor, ${ }^{2}$ Research Scholar \\ KG College of Arts and Science, Coimbatore, Tamil Nadu, India
}

\begin{abstract}
The author defines the complementary dominating set and its number in intutionistic fuzzy graph. The author discussed the order and enclave is obtained for some standard intutionistic fuzzy graph is derived.
\end{abstract}

Keyword: Intutionistic fuzzy graph, degree, complementary nil dominating set, effective degree.

\section{INTRODUCTION}

In 1999, Atanassov[1] introduced the concept of intutionistic fuzzy relation and intutionistic fuzzy graphs. Parvathi and Karunambigai [5] introduced the concept of intutionistic fuzzy graph and analyzed its components. Nagoor Gani and Sajitha Begum [11] define degree, order and size in intutionistic fuzzy graph and derive its some of their properties. Somasundaram [9] introduced the concept of domination in intutionistic fuzzy graph. Parvathi and Thamizhendhi [6] introduced the concept of domination number in intutionistic fuzzy graph. Tamizh Chelvam [10] introduced and analyzed the complementary nil dominating set in the crisp graph. The properties of nil complementary dominating graph in intutionistic fuzzy graph is discussed in this paper.

\section{Preliminaries}

\section{Definition 2.1}

A fuzzy graph $G=(\sigma, \mu)$ is a pair of functions $\sigma: V \rightarrow[0,1]$ and $\mu: V \times V \rightarrow[0,1]$, where for all $u, v \in V$ $\mu(u, v)=\sigma(u) \wedge \sigma(v)$.

\section{Definition 2.2}

Let $G=(V, E)$ be an intutionistic fuzzy graph, such that

(i) $V=\left\{v_{1}, v_{2} \ldots \ldots v_{n}\right\}$ such that $\mu_{1}: V \rightarrow[0,1]$, $v_{1}: V \rightarrow[0,1]$ denote the degree of membership and non-membership of the element $v_{i} \in V$ respectively and $0 \leq \mu_{1}\left(v_{i}\right)+v_{1}\left(v_{i}\right) \leq 1$ for every $v_{i} \in V,(\mathrm{i}=1,2, \ldots \ldots . \mathrm{n})$.

(ii) $E \subseteq V \times V \quad$ where

$v_{2}: V \times V \rightarrow[0,1]$ are $\mu_{2}: V \times V \rightarrow[0,1]$ and $\mu_{2}\left(v_{i}, v_{j}\right) \leq \mu_{1}\left(v_{i}\right) \wedge \mu_{1}\left(v_{j}\right)$ such that $v_{2}\left(v_{i}, v_{j}\right) \leq v_{1}\left(v_{i}\right) \wedge v_{1}\left(v_{j}\right) \quad$ respectively and $0 \leq \mu_{2}\left(v_{i}, v_{j}\right)+v_{2}\left(v_{i}, v_{j}\right) \leq 1$

\section{Definition 2.3}

Let $G=(V, E)$, be an intutionistic fuzzy graph. The cardinality of $\mathrm{G}$ is defined to be

$$
|G|=\left|\sum_{v_{i} \in V} \frac{1+\mu_{1}\left(v_{i}\right)-v_{1}\left(v_{i}\right)}{2}+\sum_{v_{i} \in V} \frac{1+\mu_{2}\left(e_{i j}\right)-v_{2}\left(e_{i j}\right)}{2}\right|
$$

The vertex cardinality is defined as

$$
|V|=\sum_{v_{i} \in V} \frac{1+\mu_{1}\left(v_{i}\right)-v_{1}\left(v_{i}\right)}{2}
$$

The edge cardinality is defined as

$$
|E|=\sum_{e_{i j} \in V} \frac{1+\mu_{2}\left(e_{i j}\right)-v_{2}\left(e_{i j}\right)}{2}
$$

\section{Definition 2.4}

Let $G=(V, E)$ be an intutionistic fuzzy graph. The degree of a vertex $v_{i}$ is defined by $d\left(v_{i}\right)=\left(d_{\mu}\left(v_{i}\right), d_{v}\left(v_{i}\right)\right) \quad$ where $\quad d_{\mu}(v)=\sum_{i \neq j} \mu_{i j} \quad$ and $d_{v}(v)=\sum_{i \neq j} v_{i j}$

\section{Definition 2.5}

The effective degree of a vertex $\mathrm{v}$ in intutionistic fuzzy graph, $G=(V, E)$ is defined to be the sum of the 
strong edge incident at v. It is denoted by $\delta_{E}(G)$ and $\Delta_{E}(G)$.

The minimum degree of $\mathrm{G}$ is $\delta_{E}(G)=\min \left(d_{E}(v) / v \in V\right)$.

The maximum degree of $\mathrm{G}$ is $\Delta_{E}(G)=\max \left(d_{E}(v) / v \in V\right)$

Two vertices $v_{i}$ and $v_{j}$ are said to be neighbourhood in intutionistic fuzzy graph if there is a strong edges between $v_{i}$ and $v_{j}$.

\section{Definition 2.6}

An intutionistic fuzzy graph $H^{\prime}=\left(V^{\prime}, E^{\prime}\right)$ is said to be an intutionistic fuzzy subgraph of $G=(V, E)$ if $V^{\prime} \subseteq V$ and $E^{\prime} \subseteq E$. That is $\mu_{1 i}^{\prime} \leq \mu_{1 i} ; v_{1 i}^{\prime} \leq v_{1 i}$ and $\mu_{2 i j}^{\prime} \leq \mu_{2 i j} ; \gamma_{2 i j}^{\prime} \leq \gamma_{2 i j}$ for every $\mathrm{i}, \mathrm{j}=1,2, \ldots . . \mathrm{n}$

\section{Definition 2.7}

Let $G=(V, E)$,be an intutionistic fuzzy graph. The complement of an intutionistic fuzzy graph is, denoted by $G=(\bar{V}, \bar{E})$, to be satisfied the following conditions.

(i) $\bar{V}=v$

(ii) $\overline{\mu_{1 i}}=\mu_{1 i}$ and $\overline{v_{1 i}}=v_{1 i}$ for all $\mathrm{i}=1,2, \ldots . \mathrm{n}$

(iii) $\overline{\mu_{2 i j}}=\min \left(\mu_{1 i}, \mu_{1 j}\right)-\mu_{2 i j}$ and $\overline{v_{2 i j}}=\min \left(v_{1 i}, v_{1 j}\right)-v_{2 i j}$ for all $i, j=1,2, \ldots n$

\section{Definition 2.8}

Let $G=(V, E)$ be an intutionistic fuzzy graph. A set $S \subset V$ is said to be a nil complementary dominating set of an intutionistic fuzzy graph of $G$, if $S$ is a dominating set and its complement V-S is not a dominating set. The minimum scalar cardinality over all nil complementary dominating set is called a nil complementary domination number and it is denoted by $\gamma_{\text {ncd }}$, the corresponding minimum nil complementary dominating set is denote by $\gamma_{n c d}$-set.

\section{Definition 2.9}

Let $S \subset V$ in the connected intutionistic fuzzy graph $G=(V, E)$. A vertex $u \in S$ is said to be an enclave of $\mathrm{S} \quad$ if $\quad \mu_{2}(u, v)<\max \left[\mu_{1}(u), \mu_{1}(v)\right] \quad$ and $v_{2}(u, v)<\min \left[v_{1}(u), v_{1}(v)\right] \quad$ for $\quad$ all $v \in V-S$. $N(u) \subseteq S$.

\section{Nil complementary in IFG}

\section{Theorem 3.1}

A dominating set $\mathrm{S}$ is a nil complementary dominating set if and only if it contains at least one enclave.

\section{Proof}

Let $\mathrm{S}$ be a nil complementary dominating set of a intutionistic fuzzy graph $G=(V, E)$. The V-S is not a dominating set which implies that there exit a vertex $u \in S$ such that $\mu_{2}(u, v)<\max \left[\mu_{1}(u), \mu_{1}(v)\right] \quad$ and $v_{2}(u, v)<\min \left[v_{1}(u), v_{1}(v)\right]$ for all $v \in V-S$. Therefore $u$ is an enclave of S. Hence $\mathrm{S}$ contain atleast one enclave.

Conversely, suppose the dominating set $\mathrm{S}$ contains enclaves. Without loss of generality let us take $u$ be the enclave of S. (i.e) $\mu_{2}(u, v)<\max \left[\mu_{1}(u), \mu_{1}(v)\right]$ and $v_{2}(u, v)<\min \left[v_{1}(u), v_{1}(v)\right]$ for all $v \in V-S$. Hence V-S is not a dominating set. Hence dominating set $S$ is nil complementary dominating set.

\section{Theorem 3.2}

If $S$ is a nil complementary dominating set of an intutionistic fuzzy graph $G=(V, E)$, then there is a vertex $u \in S$ such that $S-\{u\}$ is a dominating set.

\section{Proof}

Let $S$ be a nil complementary dominating set. since by theorem 3.1, every nil complementary dominating set if and only if it contains atleast one enclave. Let $u \in S$ be an enclave of s. Then $\mu_{2}(u, v)<\max \left[\mu_{1}(u), \mu_{1}(v)\right]$ and $v_{2}(u, v)<\min \left[v_{1}(u), v_{1}(v)\right]$ for all $v \in V-S$. Since $G$ is connected intutionistic fuzzy graph, there exist atleast a vertex $w \in S$ such that $\mu_{2}(u, v)=\max \left[\mu_{1}(u), \mu_{1}(v)\right]$ and $v_{2}(u, v)=\min \left[v_{1}(u), v_{1}(v)\right]$ hence $S-\{u\}$ is a dominating set.

\section{Theorem 3.3}

A nil complementary dominating set in an intutionistic fuzzy graph $G=(V, E)$ is not a singleton.

\section{Proof}

Let $\mathrm{S}$ be a nil complementary dominating set. Since by theorem 3.1, every nil complementary dominating set if and only if it contains atleast one enclave. Let $u \in S$ be an enclave of $\mathrm{s}$. Then $\mu_{2}(u, v)<\max \left[\mu_{1}(u), \mu_{1}(v)\right]$ 
$v_{2}(u, v)<\min \left[v_{1}(u), v_{1}(v)\right]$ for all $v \in V-S$. Suppose $\mathrm{S}$ contains only one vertex $u$, then it must be isolated in G. This is contradiction to connectedness. Hence nil complementary dominating set contains more than one vertex.

\section{Conclusion}

The nil complementary dominating set and its number in intutionistic fuzzy graph is defined. Some of the properties are derived in this paper. The future work will be carried on its application in social network.

\section{References}

1. Atanassov. K. T. Intutionistic fuzzy sets: Theory and its Applications. Physica, New York, 1999.

2. Bhattacharya, P. Some remarks on fuzzy graphs, Pattern Recognition Letter 5: 297-302, 1987.

3. Harary, F., Graph Theory, Addition Wesley, Third Printing, October 1972.

4. C. V. R. Harinarayanan and P. Muthuraj and P. J. Jayalakshmi, "Total strong (weak) domination intutionistic fuzzy graph", Advances in theoretical and applied mathematics, 11 (2016), 203-212.

5. M. G. Karunambigai and R. Parvathi, "Intuitionisitc Fuzzy Graph", Proceedings of $9^{\text {th }}$ Fuzzy Days International Conference Intelligence,
Advances in soft computing: Computatinal intelligence; Theory and Applications, SpringerVerlag, 20 (2006), 139-150

6. M. G. Karunambigai and R. Parvathi and R. Buvaneswari, "Constant intutionistic fuzzy graphs", Notes on Intuitionistic Fuzzy system, 17 (2011), 1, 37-47.

7. M. G. Karunambigai and R. Parvathi and R. Buvaneswari, "Arcs in intutionistic fuzzy graph", Notes on Intuitionistic Fuzzy system, 17 (2011).

8. M. G. Karunambigai and Muhammad Akram and R. Buvaneswari, "Strong and super strong vertices in intutionistic fuzzy graphs", Journals on intuitionistic fuzzy system, 30 (2016), 671-678.

9. Somasundaram, A and Somasundaram, S., Domination in Fuzzy graph-I, Patter Recognition Letter 19(9), 1998, 787-791.

10. Tamizh Chelvam. T and Robinson Chelladuari. S, Complementary nil domination number of a graph, Tamkang Journal of Mathematics, Vol.40, No.2 (2009), 165-172. Received: June

11. Nagoor Gani. A and Shajitha Begum. S, Degree, Order and Size in Intuitionistic Fuzzy Graphs, International Journal of Algorithms, Computing and Mathematics, (3) 3 (2010). 